\title{
Malignes Melanom im Bestrahlungsfeld kurz nach Radiotherapie wegen Morbus Hodgkin
}

\section{Malignant Melanoma Arising in a Radiotherapy Field Shortly After Radiation in a Patient with Morbus Hodgkin}

\author{
Autoren \\ L. Kowalzick' ${ }^{1}$, L. Eickenscheidt ${ }^{1}$, B. Schell ${ }^{1}$, E. Schaarschmidt ${ }^{2}$, T. Barthel ${ }^{3}$, D. Baaske ${ }^{3}$ \\ Institute \\ Klinik für Hautkrankheiten und Allergologie (Chefarzt: Prof. Dr.med.habil. L. Kowalzick), \\ Medizinisches Versorgungszentrum (Leitender Arzt: Dr. med. Mathias Gruber), \\ HELIOS Vogtland-Klinikum Plauen GmbH (Ärztlicher Direktor: Prof. Dr. med. habil. L. Kowalzick) \\ Klinik für Radioonkologie (Chefarzt: Dr. med. D. Baaske), Klinikum Chemnitz gGmbH \\ (Medizinischer Geschäftsführer: Prof. Dr. med. J. Klingelhöfer)
}

\section{Bibliografie}

Dol $10.1055 / \mathrm{s}-0028-1119461$

Akt Dermatol 2009; 35:

99-102 @ Georg Thieme

Verlag KG Stuttgart · New York ISSN 0340-2541

Korrespondenzadresse

Prof. Dr. med. habil.

Lutz Kowalzick

Klinik für Hautkrankheiten und Allergologie

HELIOS Vogtland-Klinikum

Plauen $\mathrm{GmbH}$

Postfach 100153

08505 Plauen

\section{Zusammenfassung \\ $\nabla$}

Wir berichten über einen 30-jährigen Patienten, der vier Monate nach Strahlentherapie von mediastinalen Lymphomen bei Morbus Hodgkin (Stadium IV b) und vorausgegangener BACOPP-D (Bleomycin, Doxyrubicin, Cyclophosphamid, Vincristin, Procarbazin, Prednisolon und Dacarbazin)-Polychemotherapie im Bestrahlungsfeld ein

\section{Einleitung \\ $\nabla$}

Im Gegensatz zum häufigeren Auftreten epidermaler Hauttumoren wie Basaliomen und Spinaliomen in Strahlentherapie-Feldern, meist nach Jahrzehnten, ist das Auftreten von malignen Melanomen hierin ein bislang selten beschriebenes Ereignis. Wir berichten hier über einen Fall des Auftreten eines malignen Melanoms im Bestrahlungsfeld nur 4 Monate nach Abschluss einer Strahlentherapie wegen eines fortgeschrittenen Morbus Hodgkin, ordnen den Fall in die bisherige Literatur ein und diskutieren mögliche Zusammenhänge zwischen Primärtumorerkrankung, Strahlentherapie und sekundärer Melanomerkrankung.

\section{Kasuistik \\ $\nabla$}

Wir berichten über einen 30-jährigen Patienten, der an einem Morbus Hodgkin Stadium IV b mit Lymphomen im oberen Mediastinum, Wirbelkörperbefall $\mathrm{C7}$ bis Th5 sowie Periduralinfiltration C7 bis Th2 erkrankt war. Von Februar bis August 2007 erfolgte eine Polychemotherapie nach dem BACOPP-D-Schema (Bleomycin, Doxorubicin, Cyclophosphamid, Vincristin, Procarbazin, Prednisolon und Dacarbazin) über 8 Zyklen. Danach waren nur noch mediastinale Restlymphome nachweisbar, sodass anschließend eine konsoli- malignes Melanom (Stadium Ia) entwickelte. Daneben fanden sich Vitiligo-Herde und ein HaloNävus am sonstigen Integument. Der mögliche Zusammenhang zwischen der Entstehung eines malignen Melanoms und einer Strahlentherapie wird anhand der aktuellen Literatur diskutiert. Die regelmäßige Kontrolle des Bestrahlungsfeldes auf auffällige Hautveränderungen wird empfohlen.

dierende Nachbestrahlungstherapie von August bis September 2007 mit 30 Gy (Einzeldosen von jeweils 2 Gy) u.a. im Bereich des oberen Thorax über opponierende Felder erfolgte ( $\bullet$ Abb.1). Etwa vier Monate später bemerkte die Ehefrau des Patienten das Neuentstehen einer pigmentierten, langsam wachsenden Hautveränderung im posterioren Bestrahlungsfeld links paravertebral in Höhe etwa des vierten Brustwirbelkörpers. Zum Zeitpunkt der Vorstellung beim Dermatologen knapp ein halbes Jahr später fand sich ein pigmentierter Tumor von ca. $4 \mathrm{~mm}$ Durchmesser mit klinischen Suspizien. Der Herd wurde als suspekter Nävuszellnävus durch Exzisionsbiopsie in toto entfernt. Die histologische Aufarbeitung ergab ein superfiziell spreitendes malignes Melanom, Invasionslevel II nach Clark mit einer Eindringtiefe nach Breslow von $0,35 \mathrm{~mm}$ ohne Mikroulzeration. Typische klinische Zeichen eines Radioderms, etwa Pigmentverschiebungen, Poikilodermie oder Teleangiektasien fanden sich nicht. Nach ca. 4 Monaten ist bei einer Photonenbestrahlung mit 30 Gy Herddosis an der Haut makroskopisch kaum etwas nachweisbar, da an der Hautoberfläche nur ca. $20 \mathrm{~Gy}$ wirksam sind. Auch histologisch ergab sich kein Anhalt für Strahlendermatitis. Bereits vor Diagnose der hämatologischen Erkrankung traten bei dem Patienten im Sommer 2006 erstmals Vitiligoherde am rechten Unterschenkel, an Rücken und Brust auf. Bei der Untersuchung fand 

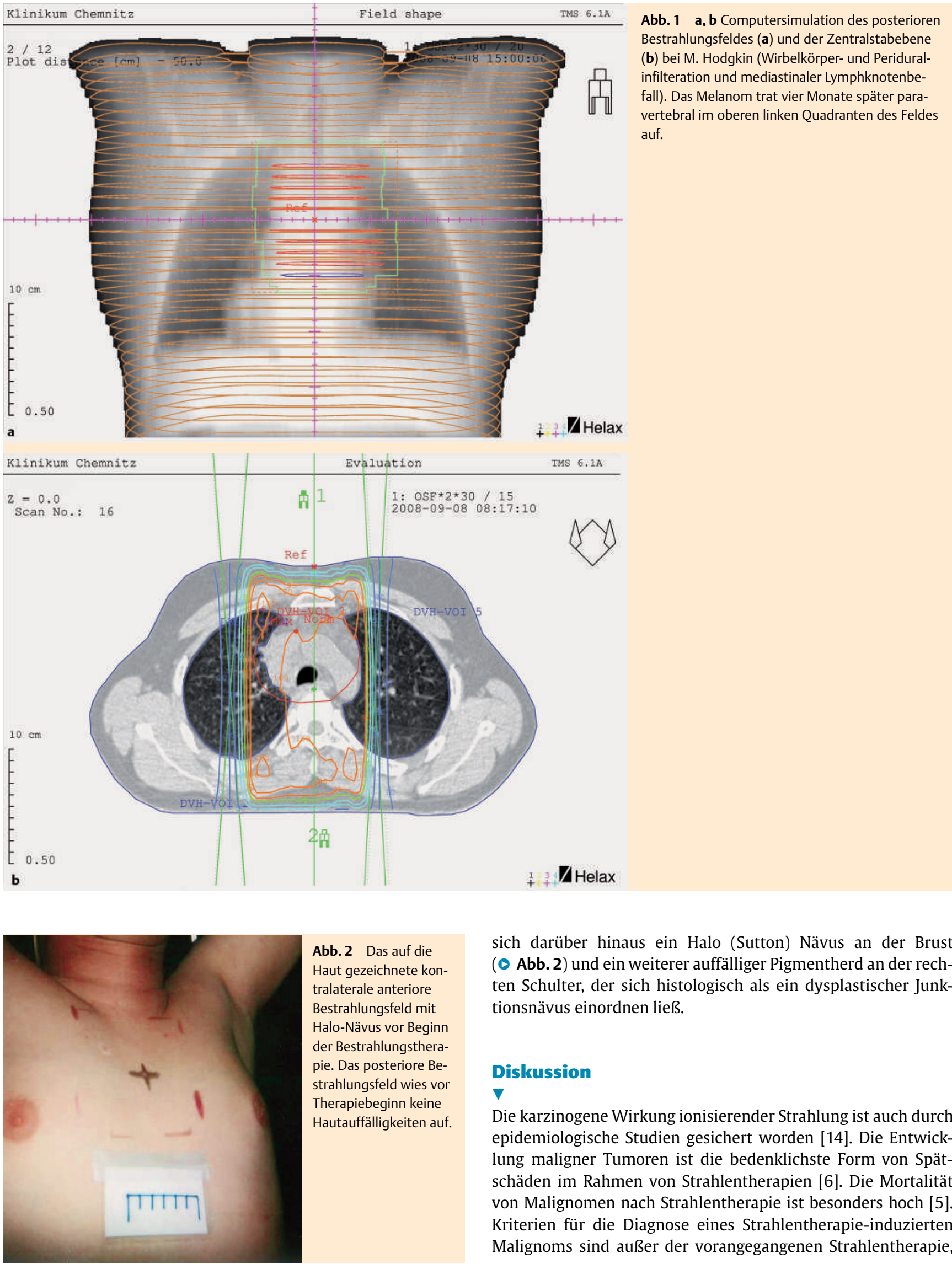

Abb. 2 Das auf die Haut gezeichnete kontralaterale anteriore Bestrahlungsfeld mit Halo-Nävus vor Beginn der Bestrahlungstherapie. Das posteriore Bestrahlungsfeld wies vor Therapiebeginn keine Hautauffälligkeiten auf. sich darüber hinaus ein Halo (Sutton) Nävus an der Brust ( Abb. 2) und ein weiterer auffälliger Pigmentherd an der rechten Schulter, der sich histologisch als ein dysplastischer Junktionsnävus einordnen ließ.

\section{Diskussion}

$\nabla$

Die karzinogene Wirkung ionisierender Strahlung ist auch durch epidemiologische Studien gesichert worden [14]. Die Entwicklung maligner Tumoren ist die bedenklichste Form von Spätschäden im Rahmen von Strahlentherapien [6]. Die Mortalität von Malignomen nach Strahlentherapie ist besonders hoch [5]. Kriterien für die Diagnose eines Strahlentherapie-induzierten Malignoms sind außer der vorangegangenen Strahlentherapie, 
Tab. 1 Publizierte Kasuistiken von 23 Fällen mit Melanomentstehung im Bestrahlungsfeld nach Strahlentherapie mit Patienten- und Therapiecharakteristika sowie beobachteten Latenzzeiten (n. a. = nicht angegeben).

\begin{tabular}{|c|c|c|c|c|c|c|}
\hline Fall Nr. & Primärtumor & $\begin{array}{l}\text { Patientenalter bei } \\
\text { Melanomdiagnose } \\
\text { (Jahre) }\end{array}$ & $\begin{array}{l}\text { Chemo- } \\
\text { therapie } \\
\text { (ja/nein) }\end{array}$ & Strahlendosis (Gy) & $\begin{array}{l}\text { Latenzzeit } \\
\text { (Jahre) }\end{array}$ & Literatur (Jahr) \\
\hline 1 & Spinaliom & 34 & $n$ & n.a. & 5 & Conley (70) \\
\hline 2 & $\begin{array}{l}\text { kongenitaler Riesen- } \\
\text { Nävuszellnävus }\end{array}$ & 78 & $\mathrm{n}$ & 50 & 10 & Conley (70) \\
\hline 3 & Keloid & 67 & $\mathrm{n}$ & n. a. & 45 & Conley (70) \\
\hline 4 & Mamma-Karzinom & 52 & $\mathrm{n}$ & 36 & 2 & Bartal (80) \\
\hline 5 & Hodgkin Lymphom & 34 & $n$ & 40 & 4 & Bartal (80) \\
\hline 6 & Hodgkin Lymphom & 25 & $\mathrm{n}$ & n. a. & 1,3 & Nelson (81) \\
\hline 7 & Synovial-Karzinom & 32 & $\mathrm{n}$ & 22 (Neutronen) & 0,75 & Waxler (84) \\
\hline 8 & (Lichen planus) & 47 & $\mathrm{n}$ & 8 & 35 & Kalemeris (85) \\
\hline 9 & Hämangiom & 47 & $\mathrm{n}$ & n.a. & 30 & Preaux (86) \\
\hline 10 & Endometrium-Karzinom & 75 & $\mathrm{n}$ & 50 & 10 & Inbar (88) \\
\hline 11 bis 15 & Kutanes T-Zell Lymphom & 20 bis 61 & $n$ & 36 (Elektronen) & 1 bis 8 & Licata (95) \\
\hline 16 bis 19 & $\begin{array}{l}2 \times \text { Hodgkin Lymphom, } \\
2 \times \text { Hirntumor }\end{array}$ & 12 bis 49 & n.a. & $35-50$ & 2 bis 29 & Corpron (96) \\
\hline 20 & Fibrosarkom & 47 & n.a. & 50 & 6 & Miracco (00) \\
\hline 21 & Ewing Sarkom & 64 & j & 64 & 5 bis 6 & Trefzer (03) \\
\hline 22 & Hämangiom & 44 & $\mathrm{n}$ & n.a. & 44 & Trefzer (03) \\
\hline 23 & Hodgkin Lymphom & 30 & j & 30 & 0,33 & aktueller Fall (08) \\
\hline
\end{tabular}

die Entstehung des Tumors im Strahlenfeld, eine hohe Strahlungsdosis und eine „lange“ Latenzzeit zwischen Strahlentherapie und Entstehung des Tumors [2]. Das beobachtete Zeitintervall zwischen der Strahlentherapie und der Entstehung von Nicht-Melanom-Hautkrebs wird mit zwischen 2 und 47 Jahren angegeben [Lit. in 7]. Bis zu einem Drittel von 202 Patienten mit chronischer Strahlendermatitis entwickelten epidermale Malignome wie Basaliom, Spinaliom oder Morbus Bowen, jedoch keine Melanome [18]. Nur wenige Berichte über das Auftreten von malignen Melanomen nach Strahlentherapie liegen vor $[1,4,7,9,10-13,16,17](\bullet$ Tab. 1$)$.

In der Mehrzahl dieser Fälle (14/23) lag die Latenzzeit unter 10 Jahren, in 4 Fällen unter zwei Jahren. Der hier beschriebene Fall weist mit einer Latenzzeit von 4 Monaten den geringsten bisher beschriebenen Zeitabstand zwischen dem Ende der Strahlentherapie und dem Neuauftreten eines malignen Melanoms auf. Die Mehrzahl der beschriebenen Melanomfälle nach Radiatio erfüllt also nicht das Kriterium einer „langen“ Latenzzeit und somit die Definition eines Strahlentherapie-induzierten Malignoms. Denkbar wäre aber, dass die Onkogenese durch ionisierende Strahlen beim Melanom anderen zeitlichen Mechanismen als denen bei epithelialen und epidermalen Tumoren folgt. Alternativ wäre möglich, dass - insbesondere bei extrem kurzer Latenzzeit, wie in unserem Fall - eine Strahlentherapie-induzierte lokale Immunsuppression das Wachstum und klinische Sichtbarwerden eines bereits subklinisch präexistenten Melanoms begünstigt. Für eine solche Annahme könnte sprechen, dass bei unserem Patienten Sutton-Nävi und Vitiligoherde bereits vor der Strahlentherapie bestanden, die ja einem klinisch diagnostizierbaren malignen Melanom vorhergehen können [15]. Eine zusätzliche immunsuppressive Wirkung hat auch die vor der Radiatio durchgeführte Polychemotherapie gehabt.

Denkbar wäre schließlich auch, dass die Lokalisation von Melanomen als Zweittumoren im Bestrahlungsfeld zufällig ist, da beispielsweise beim $M$. Hodgkin eine generell erhöhte Inzidenz von Melanomen als Zweittumor auch ohne Bezug zu Bestrah- lungsfeldern bekannt ist [8]. Aufgrund der relativ kleinen Fallzahl berichteter kutaner Melanome in Strahlentherapiefeldern können gegenwärtig keine allgemeingültigen Schlüsse gezogen werden, ob ein kausaler Zusammenhang zwischen beiden Ereignissen bestehen kann, und welche Faktoren (z. B. Art des Primärtumors, Strahlungsart, Strahlungsdosis, begleitende Chemotherapie, Patientenalter) eventuell risikoerhöhend sein könnten. Langzeit-Überwachungen von Patienten nach Strahlentherapie auch im Hinblick auf das Auftreten von Hautveränderungen im Bestrahlungsfeld sind aber zu empfehlen.

\section{Abstract}

\section{Malignant Melanoma Arising in a Radiotherapy Field Shortly After Radiation in a Patient with Morbus Hodgkin}

We report on a 30-years old male caucasian patient who developed a malignant melanoma (stage I a) in the radiotherapy field within four months after radiotherapy because of mediastinal lymphomas of Hodgkin's disease (stage IV b) and additional BACOPP-D (bleomycin, doxorubicin, cyclophosphamide, vincristine, procarbazine, prednisolone and dacarbazine) polychemotherapy in sequence. Beside of this, vitiligo lesions and a Sutton's nevus were present in other areas of the body. A possible causal connection between developement of melanoma and radiotherapy is discussed in the light of recent literature. The regular examination of radiation fields for skin changes is recommended. 


\section{Literatur}

1 Bartal AH, Cohen Y, Robinson E. Malignant melanoma arising at tattoo sites used for radiotherapy field marking. Btit J Radiol 1980; 53: $913-$ 914

2 Chudecki B. Radiation cancer of the thoracic oesophagus. Br J Radiol 1972; 45: 303-304

3 Conley J. Irradiation as an etiologic factor in the development of melanoma. Arch Otolaryng 1970; 92: 627-631

4 Corpron CA, Black CT, Ross MI et al. Melanoma as a second malignant neoplasm after childhood cancer. Am J Surg 1996; 172: 459- 462

5 Dolan R, Vaughan C, Fuleihan N. Metachronous cancer: prognostic factors including prior irradiation. Otolaryngol Head Neck Surg 1998; 119: 619-623

6 Dutrillaux B. Radiation-induced cancers. Cancer Radiother 1998; 2: $541-548$

7 Inbar M, Matzkin H, Rozin RR et al. Malignant melanoma developing in a radiation field. Brit J Radiol 1988; 61: 519-520

8 van Leeuwen FE, Klokman WJ, Hagenbeek A. Second cancer risk following Hodgkin"s disease: 20-year follow-up study. J Clin Oncol 1994; 12: $312-315$

9 Licata AG, Wilson LD, Braverman IM. Malignant melanoma and other second cutaneous malignancies in cutaneous T-cell lymphoma. Arch Dematol 1995; 131: $432-435$
10 Kalemeris GC, Rosenfeld L, Gray GF et al. Malignant melanoma of the tongue following low-dose radiation. Arch Pathol Lab Med 1985; 109: $290-291$

11 Miracco C, Materno M, De Santi MM. Unusual second malignacies following radiation therapy: subcutaneous pleomorphic rhabdomyosarcoma and cutaneous melanoma. Two case reports. J Cutan Pathol 2000; 27: 419-422

12 Nelson DF, Cooper S, Weston MG et al. Second malignant neoplasms in patients treated for Hodgkińs disease with radiotherapy or radiotherapy and chemotherapy. Cancer 1981; 48: 2386-2393

13 Préaux J, Noury-Duperrat G, Belaïch S. Épithélioma baso-cellulaire et mélanome malin apparus sur radiodermite. Ann Dermatol Venereol 1986; 113: 1011 - 1013

14 Ron E. Ionizing radiation and cancer risk: evidence from epidemiology. Radiat Res 1998; 150: 30-41

15 Schallreuther KU, Levenig C, Berger J. Vitiligo and cutaneous melanoma. A case study. Dermatologica 1991; 183: 230-245

16 Trefzer $U$, Voit $C$, Milling A et al. Malignant melanoma arising in a radiotherapy field: report of two cases and review of the literature. Dermatology 2003; 206: 265 - 268

17 Waxler $B$. Malignant melanoma in patient who received neutron beam therapy. Lancet 1984; i: $904-905$

18 Zschunke E. Ionizing radiation - a cause of malignant melanoma? Dermatol Monatsschr 1989; 176: 69-75 